\title{
STEAM - Arts Integration Frameworks for Transdisciplinarity
}

\author{
Chantal Rodier, Mohamed Galaleldin, Justine Boudreau, Hanan Anis and Liam Peyton \\ University of Ottawa \\ crodier@uottawa.ca, mgala028@uottawa.ca, jboud030@uottawa.ca, hanis@uottawa.ca and 1peyton@uottawa.ca
}

\begin{abstract}
Whether for 21 st century skills development such as creativity, communication, and collaboration or for transdisciplinary knowledge creation leading to innovation, the integration of Arts with STEM (Science, Technology, Engineering, Mathematics) fields is gaining popularity in higher education. However, a comprehensive survey of proven methodologies to integrate Arts with STEM disciplines (referred to as STEAM) currently does not exist. This paper presents the preliminary results of a systematized literature review done to characterize the integration of arts with STEM disciplines in higher education. It also uses these findings to analyze the most recent STEAM initiative of the Faculty of Engineering at the University of Ottawa. This research finds three main rationales to integrate arts with STEM and presents the frameworks discussed in the literature to do this integration. It also examines how creativity is assessed and developed within STEAM higher education contexts. This research contributes a reference of validated arts integration and creativity frameworks which can be used to setup STEAM projects or evaluate them in relation to proven methodologies. The frameworks presented in this research can be used in classrooms and professional environments.
\end{abstract}

Keywords: $\quad$ STEAM, STEAM education, Transdisciplinarity, Arts integration, STEM, Engineering design, Teamwork, Engineering Education, Creativity.

\section{INTRODUCTION}

Whether for 21 st century skills development such as creativity, communication, and collaboration or for transdisciplinary knowledge creation leading to innovation, the integration of arts with STEM fields is gaining popularity in higher education. In order to pursue those objectives, the University of Ottawa has created STEAM environments where artists and engineers work together as equal collaborators since 2017 [1]. The lack of a comprehensive survey of proven methodologies to integrate arts with STEM disciplines is the motivation behind the current research. This paper reports the results of a systematized literature review which identifies and analyzes the current state of academic research in STEAM, by investigating terms, definitions, issues, and frameworks used to integrate Arts with STEM disciplines in higher education.

Overall, this research aims to contribute a reference of successful frameworks for arts integration and creativity that achieved positive results in STEAM implementations.

\section{BACKGROUND}

The interaction of sciences and arts has a long history; however, the term STEAM was coined in 2007, during an "Americans for the Arts-National Policy"[2] roundtable discussions. Art integration was identified as important in addressing the national need to increase students' interest in STEM fields. STEAM pedagogy subsequently appeared in the US and UK curricula, focusing on K-12 education [3]. From that point on, STEAM education evolved rapidly using a myriad of approaches and definitions leading to a lack of cohesion and clarity. Moreover, the demands, and learning objectives of STEAM higher education have diverged from those of $\mathrm{K}-12$ educational environments. We aim to clarify some of these aspects, by conducting a systematized literature review of the STEAM literature.

One of the promises of STEAM in higher education is innovation [4], and Robinson [5] establishes the link between imagination, creativity and innovation. He argues that they are three related ideas: "imagination, which is the process of bringing to mind things that are not present to our senses; creativity, which is the process of developing original ideas that have value, and innovation, which is the process of putting new ideas into practice." He goes on to state that a culture of innovation depends on cultivating these three processes, and each of which is related to the others. "Just as success in athletics depends on building physical fitness, a culture of innovation depends on our fitness at the processes of imagination and creativity." [5]

For the importance creativity represents for both arts and innovation, this paper explores creativity and arts integration frameworks in STEAM literature within post- 
secondary education. It seeks answers to the following research questions.

\section{RESEARCH QUESTIONS}

\section{RQ1: What are the key terms, definitions, and issues in STEAM higher education?}

RQ2: Which educational frameworks are used to integrate Arts and creativity with STEM disciplines in higher education?

\section{METHODOLOGY}

This research employed a Systematized Literature Review (SLR) methodology for its strengths to collect, synthesize, and comprehend a specific field of study. The SLR was conducted using PSALSAR approach [6] which includes the following six steps: Protocol (defining the research scope), Search (defining search strings and databases), Appraisal (defining literature inclusion and exclusion, and quality assessment criteria), Synthesis (extracting and categorizing the data), Analysis (narrating the result and reaching conclusion) and Reporting results (stating the procedure followed and communicating the results).

Protocol: We first designed a detailed SLR protocol containing overall strategy, databases to search, search strings, research methodology, report plan, etc. The protocol was peer reviewed to reduce the possibility of researcher bias [7].

Search: Based on our research questions, we identified a set of search keys terms and synonyms to formulate into advanced search queries. The search queries were applied to the following databases: Art Full Text, ERIC, ProQuest. Scopus, and Web of Science.

Appraisal: Our study exclusion criteria were: focus on $\mathrm{K}$ 12, not peer-reviewed and our inclusion criteria were: publication on and after 2007, papers in French and English, papers from key STEAM centers (research, education, practice) found in grey literature and influential papers published prior to 2007 found by snowballing. "Snowballing refers to using the reference list of a paper or the citations to the paper to identify additional papers"[8]. However, to ensure paper quality, we evaluated the retrieved papers against the following quality assessment checklist:

Q1. Were the research goals and methodology clearly specified?

Q2. Was the study designed to achieve these goals?

Q3. Were the findings of the paper credible?

Q4. Were findings pertinent to research questions?

We rejected papers not meeting these requirements.

Covidence software [9] was used to eliminate duplicate articles retrieved from different databases and to perform a preliminary selection based on titles, keywords, and abstracts. Mendeley citation manager [10] was used to organize retrieved full articles.

Synthesis: The data extracted was synthesized by extracting, comparing, and organizing in the following preestablished categories, to answer the research questions.

\begin{tabular}{|c|l|}
\hline Question & Data Extracted \\
\hline RQ1 & Keywords, Terms, Definitions \\
\hline RQ1 & Issues, Research Questions \\
\hline RQ2 & $\begin{array}{l}\text { Frameworks to Integrate Arts and Creativity } \\
\text { with STEM }\end{array}$ \\
\hline
\end{tabular}

Analysis: The data analysis consisted of "data reduction, data display, data comparison, conclusion drawing, and verification" [3]. Once the retrieved papers were reviewed and classified, the information collected was regrouped in the following four categories:

- Definitions

- STEAM education conceptualization

- Frameworks for creativity

- Frameworks for Art integration with STEM

Reporting: The report used a narrative form to present results, discussions, and conclusions.

\section{SLR RESULTS}

The SLR results are presented using the four categories identified in our data analysis phase: Definitions, STEAM education conceptualization, Frameworks for creativity and Frameworks for Arts integration with STEM.

\subsection{Definitions}

Table 1 lists the terms and definitions found in the literature and pertinent to our research: STEAM Education, Arts Integration, Creativity, Multidisciplinary, Interdisciplinary and Transdisciplinary approaches. 
Table 1 : Definitions

\begin{tabular}{|c|c|}
\hline Term & Definitions \\
\hline STEAM Education & $\begin{array}{l}\text { STEAM (science, technology, engineering, the arts, and mathematics) education has been taken up as a varied approach to curriculum } \\
\text { that seeks to integrate, synthesize, or otherwise combine these disciplines to cultivate inter- or transdisciplinary learning experiences. [11] }\end{array}$ \\
\hline Arts Integration & $\begin{array}{l}\text { Arts integration is an approach to teaching in which students construct and demonstrate understanding through an art form. Students engage } \\
\text { in a creative process which connects an art form and another subject area and meets evolving objectives in both. [12][13] }\end{array}$ \\
\hline \multirow[t]{3}{*}{ Creativity } & $\begin{array}{l}\text { Creativity is the process of developing original ideas that have value. There are three related ideas: imagination, which is the process of } \\
\text { bringing to mind things that are not present to our senses; creativity, which is the process of developing original ideas that have value, and } \\
\text { innovation, which is the process of putting new ideas into practice. [5] p.2 }\end{array}$ \\
\hline & The ability to bring something new into existence purposefully [14] \\
\hline & $\begin{array}{l}\text { Creativity is effective novelty. That is to say, it is doing or making something new that solves a problem or usefully changes how we act, } \\
\text { think, or feel. [15] }\end{array}$ \\
\hline \multirow[t]{2}{*}{$\begin{array}{l}\text { Multidisciplinary } \\
\text { Approach }\end{array}$} & $\begin{array}{l}\text { An approach to curriculum integration which focuses primarily on the different disciplines and the diverse perspectives they bring to } \\
\text { illustrate a topic, theme or issue. A multidisciplinary curriculum is one in which the same topic is studied from the viewpoint of more than } \\
\text { one discipline. Frequently multidisciplinary and cross-disciplinary are used as synonyms describing the aim to cross boundaries between } \\
\text { disciplines. [14] }\end{array}$ \\
\hline & Collaboration among two or more disciplines but are not merged. [3] p.34 \\
\hline \multirow[t]{6}{*}{$\begin{array}{l}\text { Interdisciplinary } \\
\text { Approach }\end{array}$} & $\begin{array}{l}\text { An approach to curriculum integration that generates an understanding of themes and ideas that cut across disciplines and of the connections } \\
\text { between different disciplines and their relationship to the real world. It normally emphasizes process and meaning rather than product and } \\
\text { content by combining contents, theories, methodologies and perspectives from two or more disciplines. [14] }\end{array}$ \\
\hline & $\begin{array}{l}\text { A mode of research by teams or individuals that integrates information, data, techniques, tools, perspectives, concepts, and/or theories from } \\
\text { two or more disciplines or bodies of specialized knowledge to advance fundamental understanding or to solve problems whose solutions } \\
\text { are beyond the scope of a single discipline or area of research practice. [16] p.14 }\end{array}$ \\
\hline & Brings several disciplines together under a common theme, but each discipline remains discrete [3] p.34 \\
\hline & $\begin{array}{l}\text { Interdisciplinary studies is a process of answering a question, solving a problem, or addressing a topic that is too broad or complex to be } \\
\text { dealt with adequately by a single discipline and draws on the disciplines with the goal of integrating their insights to construct a more } \\
\text { comprehensive understanding. [17] p.16 }\end{array}$ \\
\hline & $\begin{array}{l}\text { Interdisciplinary studies combine two or more academic disciplines or fields of study to bridge multiple bases in knowledge and expertise, } \\
\text { which provides a rich base of knowledge and vales to aid in complex decision-making by enabling new models of research capable of } \\
\text { dealing with complex problems that require multi-stakeholder interests from public-private partnerships.[18] }\end{array}$ \\
\hline & $\begin{array}{l}\text { Interdisciplinary studies are multi-faceted and impact all subject areas including natural and applied sciences, social sciences, and } \\
\text { humanities. The main goal of interdisciplinary work is integration of multiple perspective used to address the challenge of complexity [16] }\end{array}$ \\
\hline \multirow[t]{4}{*}{$\begin{array}{l}\text { Transdisciplinary } \\
\text { approach }\end{array}$} & $\begin{array}{l}\text { An approach to curriculum integration which dissolves the boundaries between the conventional disciplines and organizes teaching and } \\
\text { learning around the construction of meaning in the context of real-world problems or themes.[14] }\end{array}$ \\
\hline & $\begin{array}{l}\text { A practice or domain that rises above disciplines and dissolves their boundaries to create a new social and cognitive space } \\
\text { Transdisciplinarity, therefore, is where deep integration is achieved. [19] }\end{array}$ \\
\hline & Includes fully merged disciplines without boundaries and lessons rooted in authentic problems or inquiry [3] p.34 \\
\hline & $\begin{array}{l}\text { The application of theories, concepts, or methods across disciplines with the intent of developing an overarching synthesis. } \\
\text { Transdisciplinary approaches to curriculum are valued especially for the development of multiple perspectives that inform deliberation on } \\
\text { a problem that is relevant to a real-world context, sometimes called "wicked problems" [20] p.83 }\end{array}$ \\
\hline
\end{tabular}

\subsection{STEAM education conceptualization}

The conceptualization and issues around STEAM education have evolved over time and so did the rationales for integration of Arts with STEM.

1-STEAM for inclusion in STEM education. Initially, STEAM was conceptualized as a "response to the need to increase student interest and skills in Science, Technology, Engineering, and Mathematics (STEM) fields"[3]. STEAM quickly evolves to focus on "the importance of advancing learning in STEM disciplines: engaging minority and female students in STEM subjects, increasing interest in STEM fields, and developing skills necessary for STEM careers." [3].

\section{2-STEAM for $\mathbf{2 1}^{\text {st }}$ century skills development. As of} 2012, higher education schools introduce STEAM education despite many logistical challenges posed by the interdisciplinary nature of STEAM education and a lack of proposed implementation strategies [21][20]. During that period, the rationale for STEAM education evolved to be "for the purpose of improving student engagement, creativity, innovation, problem-solving skills, and other cognitive benefits" [3], "to improve employability skills (e.g. teamwork, communication, adaptability) necessary 
for career and economic advancement" [3] and to help "meet the needs and skills of a $21^{\text {st }}$ century workforce"[3].

3-STEAM for Transdisciplinarity. As of 2018, new conceptualizations emerge for "arts-focused approaches to STEAM as inspiring transdisciplinary social" [22] and "material possibilities where disciplines meet and coalesce"[11]. Peppler \& Wohlwend [4] state that, "The promise of STEAM approaches is that, by coupling STEM and the arts, new understandings and artifacts emerge that transcend either discipline". Costantino [23] argues that "although there is recognition that an interdisciplinary curriculum structure can facilitate university students' abilities to apply knowledge to real-world contexts (...) unless institutions pay closer attention also to transdisciplinary knowledge production, students may not be adequately prepared for the demands of the modern knowledge society"

On the other hand, it is worth noting some authors [24] argue that despite the most frequently cited argument for integrating Arts with STEM disciplines being to "bring creativity, design thinking/innovation, aesthetic inquiry, and other qualities that are somehow missing from STEM education"[3], this conceptualization of STEAM fails to recognise that there are "creativity and innovation already within the STEM fields"[11]. Root-Bernstein \& RootBernstein [15] demonstrate that creativity and a creative process are, in fact, inherent in all STEAM disciplines: "For at the level of the creative process, scientists, artists, mathematicians, composers, writers, and sculptors use a common set of what we call 'tools for thinking', including emotional feelings, visual images, bodily sensations, reproducible patterns, and analogies. And all imaginative thinkers learn to translate ideas generated by these subjective thinking tools into public languages to express their insights, which can then give rise to new ideas in other's minds." The authors also add that "learning to think creatively in one discipline therefore opens the door to understanding creative thinking in all disciplines" [15].

Furthermore, Guyotte [11] argues that arts can offer much more than creativity to STEM, "what STEAM education can offer is the potential for teachers/learners to disrupt traditional disciplinary boundaries and to collaboratively engage with topics and ideas in a critical, nuanced, and interconnected way" [22]. The author claims that such a transdisciplinary approach corresponds to what is needed to address complex contemporary problems [11].

The authors cited above demonstrate the potential and three main rationales for the integration of Arts with STEM which are: 1) Inclusion, 2) $21^{\text {st }}$ century skills development, 3) Transdisciplinarity while dissolving discipline boundaries to resolve complex problems. Next, we examine the frameworks used to integrate creativity and Arts with STEM, as presented in the literature reviewed.

\subsection{Frameworks for creativity}

Three creativity frameworks are discussed in the reviewed literature: The Four C Model of Creativity, a model which helps the classification of creativity activities and research; the Propulsion Theory of Creative Contributions, a model which identifies different types of creative contributions and qualifies (almost quantifies) their impact on the field, and finally the 13 Thinking Tools of Creative People a framework of activities for the development of creativity.

\subsubsection{The Four C Model of Creativity}

Kaufman \& Beghetto [25] present a model called 'The Four C Model of Creativity' to classify creative activity levels and progression paths between them. Their four levels of creativity are: 'Big-C', 'Pro-c', 'little-c' \& 'minic'. 'Big-C' is an eminent creativity level obtained by recognized genius like Picasso, Einstein. 'Pro-c' is a professional-level expertise achieved through effort from 'little-c' or 'mini-c' with formal or informal apprenticeship and which could lead to 'Big-C'. 'little-c' is the everyday creativity like scrapbooking, solving complex scheduling problems. And finally, 'mini-c' is the novel and personally relevant interpretations often associated with exploring new things or experiences.

We find 'The Four C Model of Creativity' helpful first in the establishment of creativity as a learnable skill with an identifiable progression and second as a tool to characterize the level of creativity involved in creative activities and outcomes. We also recognize that to achieve a progression from 'little-c' to 'Pro-c' requires a major time investment (10 thousand hours [25]), an unattainable objective within a limited timeframe such as a onesemester course.

\subsubsection{Propulsion Theory of Creative Contributions}

Sternberg, Kaufman, \& Pretz [26] introduce a model which "focuses on how a creative act can change an entire field." The 'Propulsion Theory of Creative Contributions' framework is composed of 8 types of contributions. The contributions are in turn regrouped by types of impact they have on the evolution of a field. The first four contributions 
(Replication, Redefinition, Forward incrementation, Advance Forward Incrementation) all stay within the framework of an existing paradigm. Whereas the next three types (Redirection, Reconstruction/Redirection, Reinitiation) "represent attempts to reject and replace the current paradigm". The last type of creative contribution, "Integration", represents the merging of "two diverse domains to create a new idea". In other words, 'integration' transcends the initial domains and disrupts the field, as a result of transdisciplinary knowledge creation. Table 2 presents the 8 types of creative contributions.

Table 2: Propulsion Theory of Creative Contributions

\begin{tabular}{|l|l|l|}
\hline Contribution & Description & Motion Type \\
\hline 1-Replication & $\begin{array}{l}\text { Helps solidify the current } \\
\text { state of a field }\end{array}$ & Stationary \\
\hline 2-Redefinition & $\begin{array}{l}\text { Involves a change in } \\
\text { perception as to where the } \\
\text { field is }\end{array}$ & Circular \\
\hline $\begin{array}{l}\text { 3-Forward } \\
\text { Incrementation }\end{array}$ & $\begin{array}{l}\text { Occurs when a work takes } \\
\text { the field from where it is \& } \\
\text { moves it forward in the } \\
\text { current direction }\end{array}$ & Forward \\
\hline $\begin{array}{l}\text { 4-Advanced } \\
\text { Forward }\end{array}$ & $\begin{array}{l}\text { Occurs when an idea is } \\
\text { ahead of its time }\end{array}$ & $\begin{array}{l}\text { Accelerated } \\
\text { Forward }\end{array}$ \\
\hline 5-Redirection & $\begin{array}{l}\text { Takes the field in a new } \\
\text { direction }\end{array}$ & Divergent \\
\hline 6-Reconstruction & $\begin{array}{l}\text { Takes the field backwards } \\
\text { first and then in a new } \\
\text { direction }\end{array}$ & $\begin{array}{l}\text { Backward \& } \\
\text { Divergent }\end{array}$ \\
\hline 7-Reinitiation & $\begin{array}{l}\text { Takes the field from a new } \\
\text { point (not on historical path) } \\
\text { \& in a new direction }\end{array}$ & $\begin{array}{l}\text { Reinitiate } \\
\text { Motion }\end{array}$ \\
\hline 8-Integration & $\begin{array}{l}\text { Puts together ideas formerly } \\
\text { seen as distinct, unrelated, or } \\
\text { even opposed }\end{array}$ & Convergent \\
\hline
\end{tabular}

This model provides a tool to characterize the impact of creative contributions to a field. Does a creative contribution purely solidify the current understanding in this field (confirms current paradigms)? Does it take the field in a new direction based on a reassessment of history (rejects or replaces current paradigms)? Or does it disrupt the field by integrating ideas previously seen as unrelated or contradictory (disrupts current paradigms by transdisciplinary knowledge creation)?

\subsubsection{The 13 Thinking Tools of the World's Most Creative People}

After studying the creative process of renowned creative individuals, Robert and Michèle RootBernstein [15] published "Sparks of Genius: The 13 Thinking Tools of the World's Most Creative People". They demonstrate that all "imaginative thinkers" be from science, art, mathematics or other fields, use a common set of tools for thinking creatively which include "emotional feelings, visual images, bodily sensations, reproducible patterns, and analogies". They observed that creative ideas of imaginative thinkers were "first generated by subjective thinking tools" like feelings and intuition and then translated into the language and form of their profession. "No scientist thinks in formula". The 13 Thinking Tools are:

1. Observing (use all senses),

2. Imaging (create mental images),

3. Abstracting (reveal the essence),

4. Recognizing Patterns (observe \& analyse)

5. Forming Patterns (combine $\&$ innovate)

6. Analogizing (bridge the known \& the unknown)

7. Body Thinking (use body intelligence, proprioception)

8. Empathizing (see thru other people's eyes)

9. Dimensional Thinking (alter dimensional views)

10. Modelling (simplify and give a form)

11. Playing (explore without fear or constraint)

12. Transforming (express idea in different forms)

13. Synthesizing (synthesize multimodal info)

These 13 skills are used by artists and scientists alike as either a complete or partial tool set of skills depending on the challenge at hand. We see this framework as having great potential as a framework to develop different aspects of creative minds.

\subsection{Frameworks for Arts Integration with STEM}

Three frameworks were identified in the literature for integrating arts with STEM disciplines: the Design Thinking model, a popular model at the moment; the Creative Enquiry Process model, a model which leverages art studio signature pedagogy and creativity development frameworks and a Methodological framework for transdisciplinary learning and collaborative sustainable action.

\subsubsection{Design Thinking Model}

"Design thinking as a term denotes the cognitive processes or thinking skills that designers use to do their work"[27]. There is a large variety of design thinking approaches which are used in different contexts. Design Thinking is used to integrate STEAM into engineering, is used on its own by engineers and visual artists [28]. "One of the most popular, commonly noted and well-established design thinking models is the Standard design model created within the Stanford School of Design" [29]. There are five modes in the Stanford model which are used to 
work through a problem: empathize, define, ideate, prototype and test [29].

The Design Thinking model is currently very popular in STEM and STEAM labeled activities, as evidenced by the number of papers found in this review. However, the level of arts integration in its application varies greatly: from referring to the model itself as a project management tool or an art/design tool without any other art connection, to working on design problems making extensive creative use of the ideation \& prototyping phases of the method.

Overall, however, our STEAM focused literature review brought out few examples of strong arts integration using the Design Thinking methodology. As Perignat \& KatzBuonincontro [3] synthesized, the "A" in STEAM holds different meanings for different people and this causes some confusion in the field. The A in STEAM could represent any of these three meanings: "Arts Education, Arts as any non-STEM discipline, or Arts as a synonym for project-based learning, problem-based learning, technology-based learning, or making." Since 'arts' may not even be mentioned in articles about STEAM and Design Thinking, we conclude that 'arts' was defined as a form of experiential learning in those contexts.

\subsubsection{Creative Enquiry Process Model}

Costantino [23] proposes an iterative Creative Enquiry Process model for Arts Integration with STEM. The model encompasses the essential aspects of studio-based learning: critical making (hands-on inquiry), object-based learning (contextual understanding through the study of aesthetic objects), critique (classroom acts as critique partners where students articulate their intent) and exhibition/presentation (an authentic performance assessment of learning and development through aesthetic objects, accompanied by statements of artistic intent). The model is composed of the following twelve phases presented in a circle: 1) find/frame problem, 2) multi-model inquiry/material exploration, 3) refine problem, 4) in-process critique, 5) multimodal inquiry/material exploration, 6) draft/model/design, 7) inprocess critique, 8) multi-modal inquiry, 9) draft/model/design, 10) in-process critique, 11) refine, 12) exhibition/presentation. The author also used The Thirteen Thinking Tools [15] seen earlier, as a conceptual and curricular organizing framework for their course.

The Creative Enquiry Process model is focused on leveraging the studio art signature pedagogy and creativity development frameworks to integrate Arts with STEM. It ensures that the key studio art phases (critical making, object-based learning, critique, and exhibition / presentation) are central to the model.

\subsubsection{Methodological framework for transdisciplinary}

CEEA21; Paper 131

University of PEI; June $21-23,2021$
Trott, et al. [30] make an eloquent case that most current STEAM implementations are instrumentalizing the arts for the benefits of STEM learning rather than activating "the transformative potential of the arts for social change."[30] They add that: "Ultimately, the arts are instrumentalized towards strengthening students' sustained STEM interest and engagement, thus reproducing-rather than dissolving - existing disciplinary hierarchies in the name of interdisciplinarity. More generally, the stated purpose of integrating arts and sciences is often to deepen students' content knowledge (i.e., learning "what is"), rather than to promote students' critical engagement with reality (authentic problems) and spur social transformation (i.e., asking "what if?")" [30].

The 'Methodological framework for transdisciplinary' proposed by the authors focuses on critical engagement (a "bottom-up" approach) and aims to engage the full and active participation of the 'whole person' in a transformative transdisciplinary learning experience. The action-oriented combination of 'top-down' knowledge sharing and 'bottom-up' knowledge creation processes invite participants "to learn about, connect with and act on sustainability challenges in a locally meaningful ways" [30]. More specially the framework combines 3 main elements: (1) "transdisciplinary learning" a top-down approach "centered on real-world problems" rather than centered on a discipline to acquire knowledge, along with (2) a "participatory process", a bottom-up approach which encourages "personal connections to sustainability challenges" which also "blends the boundaries" between all participants (educators, students, community members, etc.). As the project evolves elements (1) \& (2) meet in (3) "collaborative action", "the culmination of transdisciplinary learning (i.e., contemplating 'what is') and participatory process (i.e., imagining 'what if?')." The authors argue that this process is "allowing learners to both envision and enact alternative futures" [30].

Trott, et al. also argue sustainability challenges (characterized as "wicked problems") are problems needing transdisciplinary solutions. As such, sustainability challenges are good problems to tackle with the 'Methodological framework for transdisciplinary', a new art-science integration model for social change [30].

As seen above, Guyotte [11] argues that Arts have disruptive powers and Trott, et al. [30] claim their model for integrating arts with STEM offers the full disruptive power of the Arts. 


\section{SLR DISCUSSION - Arts Integration with STEM at uOttawa}

In this section, we use our research findings to analyse our latest STEAM initiative. We briefly describe the initiative and then apply our research findings to our empirical methodology.

\subsection{Latest STEAM initiative}

Since 2017, University of Ottawa has consistently brought artists and engineers to work together as equal collaborators within interdisciplinary projects. See Rodier et al. [1] for a complete account of the progressive integration of arts with STEM. Our latest initiative entitled STEAM Creation, is an interdisciplinary course where 12 students in arts and 12 in engineering conceive and design small-scale functional prototypes of interactive art installations about key environmental issues.

The course addresses the language, technologies, standards, social practices, and material-based inquiries specific to art and engineering disciplines and explores how they can mutually inform each other. Using Design Thinking combined with studio-art methodology, we covered topics and activities in: team dynamics/management, innovation, creativity, artistic creation, artistic process, self/group critique, peer learning, and client interaction. Each interdisciplinary team chose their theme and medium to answer the question: "What is the most urgent environmental problem for you?". At the end of the class, team present their prototype to prospective clients selected for their interest in investing in a summer internship for students to create a full-scale version of their prototype to install in their corporation.

\subsection{Applying the research findings to our latest STEAM initiative}

In this subsection, we use the research findings and compare them with our empirical approach for assessment and validation.

\subsubsection{STEAM education conceptualization analysis}

Using the STEAM education conceptualizations and definitions found in this research, we characterize our past initiatives for arts integration with STEM disciplines to be based on the 'STEAM for $21^{\text {st }}$ century skills development' rationale. We aimed to improve our students' creativity, innovation, and problem-solving skills to help them meet the challenges of the $21^{\text {st }}$ century work environment. However, as we acquire experience in this process and observe the students' journeys, we see teams achieve productive and transformative transdisciplinarity. In those teams, discipline boundaries disappear to the benefit of creative complex problem solving. As such, the teams reach the documented third rationale for arts integration of 'STEAM for Transdisciplinarity'. We now aim to systematically achieve Transdisciplinarity within our artsintegration projects.

We look next at the methodology used in our STEAM Creation course to compare it with this research findings and to assess its effectiveness.

\subsubsection{Arts integration frameworks analysis}

Our STEAM Creation curriculum is based on the Stanford Design Thinking 5 phase framework including: Empathize, Define, Ideate, Prototype, Test. This framework has been successfully used in our engineering design courses for a few years. While using this methodology, we introduce thinking tools associated with each phase as well as notions of artistic creation, team dynamics, team management, and innovation principles. Specific deliverables are associated with each phase.

We integrate artistic creation notions into the course curriculum by enhancing the basic Design Thinking framework with signature pedagogies used in studio-art courses. We add critical making, object-based learning, prototyping, peer critiques and exhibition of final projects, creating a uOttawa STEAM framework.

Comparing our approach of integrating studio-art pedagogies to the Design Thinking model to our research, we conclude that our approach resembles Costantino's [23] Creative Enquiry Process Model, which was successfully used in an art-engineering student team context very similar to ours.

Moreover, the way we asked our students to approach the challenges presented by key environmental issues is similar to Trott, et al. [30] 'Methodological framework for transdisciplinary'. We used a combination of 'bottom-up' and 'top-down' approaches with our slightly disoriented students. We ask each team member to select a skill they had which would serve the project and to teach it to teammates. The skill they chose had to relate to their knowledge base, abilities and to the environmental issue selected by the team. Adding to the challenge openness and to our students' disorientation, they had complete technical and artistic freedom to choose the approach to their chosen 
key environmental issue (any technique and any art form). We supplemented this 'bottom-up' strategy with key readings and in-class presentations for sharing knowledge in a more traditional 'top-down' approach.

Overall, our uOttawa STEAM framework combined with a 'bottom-up' and 'top-down' content strategy was successful in addressing our course objectives. And according to our research findings, our approach followed validated frameworks for Transdisciplinarity.

The analysis of student blogs shows a higher level of overall satisfaction from engineering students than arts students. However, we believe our course curriculum was too content and deliverable heavy. It did not leave enough team exploration, play and reflection time (in-class \& within the schedule) as Costantino's [23] model suggests with two "multi-model inquiry/material exploration" stages to do so. Consequently, our teams were focused on a steady stream of deliverables which reduced time for team creative exploration. Fewer deliverables are envisioned for our next course iteration.

\subsubsection{Creativity frameworks analysis}

Moreover, our course aimed at further developing participants' creativity through a series of activities. When we analyzed our activities with the creativity frameworks found in this SLR, we concluded that our course activities corresponded to six of Root-Bernstein's 13 Thinking Tools for creative thinkers. We list each activity used and identify the corresponding thinking tool (TT).

1-Formal analysis. We organized a guided tour of a contemporary art exhibit at a local fine art museum where art and engineering students participated in visual formal analyses of contemporary artworks with a museum guide. Following the visit, each student had to write a formal analysis of an artwork of their choice. This activity develops objective observation and corresponds to Thinking Tool \#1 (TT\#1) - Observing. As [15] states "all knowledge begins in observation. Observation goes beyond the visual; it involves all senses. The mind must be trained to observe, and the skill of observation must be practiced."

2- Lego Serious Play. We used Lego Serious Play [31] methodology to get our interdisciplinary teams to reflect on the pressing environmental issue each team had chosen for their project. With a limited set of Lego blocks and limited time, they had to reach a block representation of their issue, present it and animate a group discussion about it using a prescribed language formulation. This activity activated abstracting abilities corresponding to TT\#3-Abstracting
"The process of revealing the critical essence of something" [15] and TT\#11-Playing. Playing is an activity which "provides a fun and risk-free means of seeing from a fresh perspective, learning without constraint, exploring without fear. Play transforms knowledge and builds understanding" [15]

3-Empathy mapping. Empathy is a key phase of Design Thinking. Students were asked to create empathy maps and personas to think about their project from different perspectives. This corresponds to TT\#8 Empathizing. "To see the world through other people's eyes." [15]

4-Build prototypes. During the semester students created two progressively more complex prototypes (from sketches to physical prototypes) of their intended final project. This activity corresponds to TT\#9 Dimensional Thinking which "involves moving from $2 \mathrm{D}$ to $3 \mathrm{D}$ or visa versa, mapping, altering the proportions of an object, or thinking beyond space and time as we know them." [15] The same activity also involves TT\#10 Modeling. "Models can only be made after a real system or situation has been observed, simplified, re-scaled and given a form whether it is physical, verbal, mathematical or artistic." [15]

The four creative activities used in our course correspond to six thinking tools. Our activities were effective, however, we made two observations. First, to improve the residual benefits of activities like observing (TT\#1) and abstracting (TT\#3), students would need to practice throughout the semester and not only for one class or for one assignment. Second, the effectiveness of activities more specific to art-pedagogy, like formal analysis and abstracting, rests on 2 elements: 1) making an explicit connection between the activity and the nonartistic targeted benefits and 2) the collaboration of art students more familiar with the type of exercises to demonstrate their effectiveness.

Considering the 'The Four C Model of Creativity', we aim for a student progression from 'little-c' towards 'Pro$\mathrm{c}^{\prime}$ in their creative skills. However, we recognize it is an ambitious objective within a one-semester course. This objective is more attainable within a program.

The 'Propulsion Theory of Creative Contributions' model could be used to assess the impact of interdisciplinarity in the course outcomes: Are the outcomes confirming typical outcome expected? or Are they more innovative and disruptive than expected due to the occurrence of transdisciplinary knowledge creation?

This STEAM Creation course was disrupted by COVID19 pandemic measures, forcing an on-line end of semester, and a rapid adaptation in team dynamics, studio 
access and final project deliverables. In this context, transdisciplinarity was not fully achieved. However, we conclude that the methodologies we used correspond to proven methodologies found in this research. This comparison to research findings grounds our empirical methodology and validates it.

\section{CONCLUSION}

The preliminary results of this systematized literature review have helped us characterize the integration of arts with STEM disciplines in higher education. We first clarified a lexicon surrounding STEAM, by defining: STEAM Education, Arts Integration, Creativity, Multidisciplinary, Interdisciplinary and Transdisciplinarity. We then uncovered STEAM conceptualizations and issues over time that led us to three main rationales for integrating arts with STEM which are: 1- STEAM for inclusion in STEM education, 2- STEAM for $21^{\text {st }}$ century skills development, 3- STEAM for Transdisciplinarity where discipline boundaries are dissolve to resolve complex problems.

Then, we studied two frameworks to assess creativity: 'The Four C Model of Creativity' [25] and the 'Propulsion Theory of Creative Contributions' [26] and a third model to develop creativity 'The 13 Thinking Tools of creative people' [15].

Next, we identified three frameworks to integrate arts with STEM. The well-known 'Design Thinking Model' [29] frequently used in STEM and in design projects. The two emerging models : 'Creative Enquiry Process Model' [23] focusing on key studio art elements (critical making, object-based learning, critique, and exhibition/presentation), and the 'Methodological framework for transdisciplinary' [30] which holds the promise of true transdisciplinary leading to disruptive innovations.

Finally, we examined our latest STEAM initiative in relation to our SLR findings to ground our empirical experience with proven academic research.

We conclude that the creativity frameworks examined can effectively be used in conjunction with arts integration frameworks and that the arts integration frameworks studied offer a progressively increasing potential for transdisciplinarity. Moreover, comparing these research results with the empirical methodology used in our latest STEAM initiative appears to validate our approach to achieve transdisciplinarity. In our next course iteration we would, however, reduce the number of deliverables and increase time for team creative exploration as suggested by Costantino's [23] "multi-model inquiry/material exploration" stages. We would also increase 'bottom-up' processes to further position our students as "radical visionaries and change agents for a sustainable future" as presented by Trott, et al. [30].

This comparison also indicates the usefulness of our research results as a reference to validated arts integration and creativity frameworks. It can be used to setup STEAM projects or evaluate them in relation to proven methodologies. The frameworks presented in this research can be used in classrooms and professional environments.

\section{Acknowledgements}

We would like to thank the Faculty of Arts and the Departments of Visual Arts, Music and Theatre for their support and participation in these programs as well as the clients for the student projects. These programs are also supported by the NSERC Chair in Entrepreneurial Engineering Design at the University of Ottawa.

\section{References}

[1] C. Rodier, M. Galaleldin, J. Boudreau, and H. Anis, "From STEM to STEAM in engineering design," Proc. Can. Eng. Educ. Assoc., 2019.

[2] A. for the Arts, "2007 - The Role of the Arts in Building the $21 \mathrm{st}$ Century American Workforce," 2007. https://www.americansforthearts.org/byprogram/reports-and-data/legislation-policy/nationalarts-policy-roundtable/2007-the-role-of-the-arts-inbuilding-the-21st-century-american-workforce.

[3] E. Perignat and J. Katz-Buonincontro, "STEAM in practice and research: An integrative literature review," Think. Ski. Creat., vol. 31, 2019.

[4] K. Peppler and K. Wohlwend, "Theorizing the nexus of STEAM practice," Arts Educ. policy Rev., vol. 119, no. 2, pp. 88-99, 2018

[5] K. Robinson and J. R. Lee, Out of our minds. Wiley Online Library, 2011.

[6] W. Mengist, T. Soromessa, and G. Legese, "Method for conducting systematic literature review and metaanalysis for environmental science research," MethodsX, vol. 7, p. 100777, 2020

[7] S. Keele, "Guidelines for performing systematic literature reviews in software engineering," Citeseer, 2007.

[8] C. Wohlin, "Guidelines for snowballing in systematic literature studies and a replication in software engineering," in Proceedings of the 18th international conference on evaluation and assessment in software engineering, 2014, pp. 1-10.

[9] Covidence, "Covidence." [Online]. Available: https://www.covidence.org.

[10] Mendeley, "Mendeley," Elsevier. [Online]. Available: 
https://www.mendeley.com.

[11] K. W. Guyotte, "Toward a Philosophy of STEAM in the Anthropocene," Educ. Philos. Theory, vol. 52, no. 7, pp. 769-779, 2020.

[12] L. B. Silverstein and S. Layne, "What is arts integration," Washington, DC Kennedy Cent. Perform. Arts, 2010

[13] C. Liao, "From Interdisciplinary to Transdisciplinary: An Arts-Integrated Approach to STEAM Education," Art Educ., vol. 69, no. 6, pp. 44-49, 2016.

[14] UNESCO, "Glossary of curriculum terminology," Internation Bureau of Education. http://www.ibe.unesco.org/en/glossary-curriculumterminology (accessed Mar. 01, 2021).

[15] R. Root-Bernstein and M. Root-Bernstein, Sparks of genius: The 13 thinking tools of the world's most creative people. Houghton Mifflin Harcourt, 2001.

[16] R. Frodeman, J. T. Klein, and R. C. D. S. Pacheco, "The Oxford Handbook of Interdisciplinarity INDEX," INTERdisciplina, vol. 1, no. 1.2015

[17] R. Szostak, "Interdisciplinary and transdisciplinary multimethod and mixed methods research," in The Oxford handbook of multimethod and mixed methods research inquiry, 2015.

[18] R. Luppicini, "Digital Transformation and Innovation Explained: A Scoping Review of an Evolving Interdisciplinary Field," in Interdisciplinary Approaches to Digital Transformation and Innovation, IGI Global, 2020, pp. 1-21.

[19] J. T. Klein, "Voices of Royaumont," Transdiscipl. Recreating Integr. Knowl., pp. 3-13, 2000.

[20] L. R. Lattuca, "Learning interdisciplinarity: Sociocultural perspectives on academic work,"J. Higher Educ., vol. 73, no. 6, pp. 711-739, 2002.

[21] T. E. Costantino, "Problem-based learning: A concrete approach to teaching aesthetics," Stud. art Educ., vol. 43, no. 3, pp. 219-231, 2002.

[22] K. W. Guyotte, N. W. Sochacka, T. E. Costantino, J. Walther, and N. N. Kellam, "STEAM as social practice: Cultivating creativity in transdisciplinary spaces," Art Educ., vol. 67, no. 6, pp. 12-19, 2014.

[23] T. E. Costantino, "STEAM by another name: Transdisciplinary practice in art and design education," Arts Educ. Policy Rev., vol. 119, no. 2, pp. 100-106, Apr. 2018

[24] S. Ghanbari, "Learning across disciplines: A collective case study of two university programs that integrate the arts with STEM.," Int. J. Educ. Arts, vol. 16, no. 7, 2015.

[25] J. C. Kaufman and R. A. Beghetto, "Beyond Big and Little: The Four C Model of Creativity," Rev. Gen. Psychol., vol. 13, no. 1, pp. 1-12, 2009, doi: 10.1037/a0013688.

[26] R. J. Sternberg, J. C. Kaufman, and J. E. Pretz, "The propulsion model of creative contributions applied to the arts and letters," J. Creat. Behav., vol. 35, no. 2, pp. 75$101,2001$.

[27] A. D. Watson, "Design thinking for life," Art Educ., vol. 68 , no. 3, pp. 12-18, 2015.

[28] G. A. Boy, "From STEM to STEAM: toward a humancentred education, creativity \& learning thinking," in Proceedings of the 31st European conference on cognitive ergonomics, 2013, pp. 1-7.

[29] D. Henriksen, R. Mehta, and S. Mehta, STEAM Education, no. January. 2019.

[30] C. D. Trott, T. L. Even, and S. M. Frame, "Merging the arts and sciences for collaborative sustainability action: a methodological framework," Sustain. Sci., vol. 15, no. 4, pp. 1067-1085, 2020.

[31] Lego, "Lego Serious Play Method," Lego, 2019. https://seriousplay.training/lego-serious-play/ (accessed Mar. 01, 2021). 